\title{
Electronic Structure of Halogen Doped $\mathrm{CuCr}_{2} \mathrm{Se}_{4}$
}

\author{
M. Liberati ${ }^{1,6}$, J. R. Neulinger ${ }^{2}$, R.V. Chopdekar ${ }^{3,1}$, J.S. Bettinger ${ }^{1}$, \\ E. Arenholz ${ }^{4}$, W.H. Butler ${ }^{5}$, A.M. Stacy ${ }^{2}$, Y.I. Idzerda ${ }^{6}$, Y. Suzuki $^{1}$ \\ ${ }^{1}$ Materials Science and Engineering, UC Berkeley, Berkeley, California; \\ ${ }^{2}$ Department of Chemistry, UC Berkeley, Berkeley, California; \\ ${ }^{3}$ School of Applied Physics, Cornell University, Ithaca, New York; \\ ${ }^{4}$ Advanced Light Source, Lawrence Berkeley National Laboratory, Berkeley, \\ California; \\ ${ }^{5}$ Department of Physics and Astronomy, University of Alabama, Tuscaloosa, \\ Alabama. \\ ${ }^{6}$ Department of Physics, Montana State University, Bozeman, Montana;
}

\begin{abstract}
We have employed element and chemically sensitive X-ray absorption spectroscopy (XAS) and X-ray magnetic circular dichroism (XMCD) in order to address a long standing controversy regarding the electronic and magnetic state of $\mathrm{CuCr}_{2} \mathrm{Se}_{4}$ via halogen doping of the Se anion site in $\mathrm{CuCr}_{2} \mathrm{Se}_{4-\mathrm{x}} \mathrm{Y}_{\mathrm{x}}(\mathrm{Y}=\mathrm{Cl}$ and $\mathrm{Br})$. Long range magnetic order is observed above room temperature for all samples. The Cr $L_{2,3}$ XAS spectra show a prevalent $3+$ valence for the $\mathrm{Cr}$ ions independent of doping concentration and doping agent. The $\mathrm{Cu} L_{2,3}$ XAS spectra show a combination of $1+$ and 2+ valence states for all samples. XMCD spectra indicate the presence of a magnetic moment associated with the $\mathrm{Cu}$ ions that is aligned antiparallel to the $\mathrm{Cr}$ moment.
\end{abstract}


$\mathrm{CuCr}_{2} \mathrm{Se}_{4}$ is a complex chalcogenide compound that has been of renewed interest because it has been theoretically predicted to be highly spin polarized (1) and exhibit significant Kerr rotation (2), thus making it a candidate material for new magnetic devices. The compound has been extensively studied in bulk form in the 60's-70's and characterized as a metallic ferromagnet with Curie temperature $\left(\mathrm{T}_{\mathrm{C}}\right)$ of approximately $440 \mathrm{~K}(3,4)$. However, the details of the electronic and magnetic state of $\mathrm{CuCr}_{2} \mathrm{Se}_{4}$ remain controversial. Two theoretical models have been proposed over the years. The first one by Lotgering (5) suggests an electronic configuration of $\mathrm{Cu}^{+}\left(\mathrm{Cr}^{3+} \mathrm{Cr}^{4+}\right) \mathrm{Se}^{2-}{ }_{4}$ where the ferromagnetism is due to the indirect coupling of the $\mathrm{Cr}^{3+}$ ions spins with the $\mathrm{Cr}^{4+}$ ones through $90^{\circ}$ Se bonds, while the $\mathrm{Cu}^{+}$ions do not contribute since they have a full $3 d$ shell. The metallicity of the system is explained in terms of hole conduction in the few empty valence band levels formed by $\mathrm{Se}^{2-} p$ and $\mathrm{Cr}^{4+} d$ states. The second model by Goodenough (6) suggests a configuration of $\mathrm{Cu}^{2+}\left(\mathrm{Cr}^{3+}\right)_{2} \mathrm{Se}^{2-}{ }_{4}$ where the overall ferromagnetism (or more correctly the ferrimagnetism) is mainly due to the contributions of two opposing magnetic moments - one associated with the super-exchange interaction of $\mathrm{Cr}^{3+}$ ions through $90^{\circ}$ Se bonds and the other with a $\mathrm{Cu}^{2+}$ delocalized hole. In this model the metallic behavior is associated with the $t_{2 g}$ orbitals of the delocalized $\mathrm{Cu}^{2+}$ hole. The main difference between the two models resides in the valence states and associated magnetic contributions of the $\mathrm{Cr}$ and $\mathrm{Cu}$ ions. To date there have been a similar number of experimental papers based on magnetization and Hall effect measurements supporting both theories $(3,4,7,8)$.

X-ray absorption spectroscopy (XAS) and its magnetic variant, X-ray magnetic circular dichroism spectroscopy (XMCD), have proved over the last 20 years to be 
exceptional experimental tools to characterize the electronic and magnetic state of the $3 d$ transition metals in complex system given their element and chemical sensitivity (9). However, these techniques have been applied only recently to $\mathrm{CuCr}_{2} \mathrm{Se}_{4}$ in two studies. The first one by Kimura et al. (10) reports an XAS-XMCD study on a $\mathrm{CuCr}_{2} \mathrm{Se}_{4}$ single crystal. They confirm a $3+$ valence for the $\mathrm{Cr}$ ions and the presence of a $\mathrm{Cu}$ magnetic moment opposite to that of $\mathrm{Cr}$, thus supporting the Goodenough model; however, they also assign a $1+$ valence to the $\mathrm{Cu}$ ions (i.e., a full $3 d$ shell) that is inconsistent with $\mathrm{Cu}$ magnetism and divalent $\mathrm{Cu}$ ions proposed in Goodenough's theory. The second study by Noh et al. (11) focuses on the XAS-XMCD of polycrystalline $\mathrm{CuCr}_{2-\mathrm{x}} \mathrm{Ti}_{\mathrm{X}} \mathrm{Se}_{4}$ in which $\mathrm{Ti}$ substitution of $\mathrm{Cr}$ is used to vary $\mathrm{Cr}$ and $\mathrm{Cu}$ valence states. Here both $1+$ and 2+ valence states are attributed to the $\mathrm{Cu}$ ions; these results are at odds with Goodenough's interpretation of the $\mathrm{Cu}$ electronic configuration.

Halogen doped spinels $\mathrm{CuCr}_{2} \mathrm{Se}_{4-\mathrm{x}} \mathrm{Y}_{\mathrm{x}}$ (with $\mathrm{Y}=\mathrm{Cl}, \mathrm{Br}$ ) also provide a model system to understand the electronic structure of $\mathrm{CuCr}_{2} \mathrm{Se}_{4}$. Halogen doping of the Se sites provides for a variation in the concentration of the $\mathrm{Cu}^{2+}$ holes, thus testing the validity of the two theories described above. The magnetic Compton effect (12) and bulk sensitive magnetometries $(13,14)$ have been used to probe the magnetism in these samples. In these studies, increasing dopant concentration gives rise to an increase of the total magnetic moment, a decrease of the $\mathrm{T}_{\mathrm{C}}$ and an increase of the lattice parameter. More specifically, Miyatani et al. have proposed an electronic configuration of $\mathrm{Cu}_{1}$ ${ }_{\mathrm{x}}{ }^{2+} \mathrm{Cu}_{\mathrm{x}}{ }^{+} \mathrm{Cr}^{3+}{ }_{2} \mathrm{Se}_{4-\mathrm{x}}{ }^{2-} \mathrm{Br}_{\mathrm{x}}^{-}$to explain their magnetization data of single crystal and polycrystalline samples (14). However the electronic configuration and associated 
magnetic contributions of halogen doped $\mathrm{CuCr}_{2} \mathrm{Se}_{4}$ have yet to be definitively determined due to the lack of element and chemical sensitivity in these studies.

In this paper we have employed element and chemically sensitive XAS and $\mathrm{XMCD}$ in order to address a long standing controversy regarding the electronic and magnetic state of $\mathrm{CuCr}_{2} \mathrm{Se}_{4}$ via halogen doping of the Se anion site in $\mathrm{CuCr}_{2} \mathrm{Se}_{4-\mathrm{x}} \mathrm{Y}_{\mathrm{X}}$ ( $\mathrm{Y}=\mathrm{Cl}$ and $\mathrm{Br}$ ). XAS spectra of $\mathrm{Cl}$ and $\mathrm{Br}$ doped $\mathrm{CuCr}_{2} \mathrm{Se}_{4}$ reveal a predominantly $\mathrm{Cr}^{3+}$ electronic configuration and a mixed $\mathrm{Cu}^{+} / \mathrm{Cu}^{2+}$ electronic configuration. XMCD spectra confirm the presence of magnetic $\mathrm{Cu}^{2+}$ ions whose moments are antiparallel to those of the $\mathrm{Cr}^{3+}$ ions. Together these results suggest that neither the Lotgering nor Goodenough model explains the electronic and magnetic structure of the $\mathrm{CuCr}_{2} \mathrm{Se}_{4}$ family completely.

All single crystals have been grown by a Chemical Vapor Transport (CVT) technique using three different transport agents $\left(\mathrm{SeBr}_{4}, \mathrm{CrCl}_{3}\right.$, and $\left.\mathrm{SeCl}_{4}\right)$ (15). The concentrations of the halogen dopants $(\mathrm{Cl}$ and $\mathrm{Br})$ substituting the Se anions have been obtained by microprobe analysis. They are $\mathrm{x}=0.0017,0.0079,0.0248,0.1273,0.2882$ and 0.3374 for $\mathrm{CuCr}_{2} \mathrm{Se}_{4-\mathrm{x}} \mathrm{Cl}_{\mathrm{x}}$ and $\mathrm{y}=0.0168,0.1369,0.2121$ and 0.3932 for $\mathrm{CuCr}_{2} \mathrm{Se}_{4-\mathrm{y}} \mathrm{Br}_{\mathrm{y}}$. Structural characterization by X-ray diffraction has shown a trigonally distorted structure compared to the usual cubic spinel structure (15) independent of the halogen doping agent. Magnetic measurements of the overall magnetic moment, $\mathrm{T}_{\mathrm{C}}$ and hysteresis loops have been performed on a Vibrating Sample Magnetometer (VSM) and Superconducting Quantum Interference Device (SQUID) magnetometer. XAS and XMCD measurements of the $\mathrm{Cr}$ and $\mathrm{Cu} L_{2,3}$ edges have been performed at the Advanced Light Source using an elliptical polarized undulator beamline (16). A normal incidence configuration, i.e. the photon beam impinging on the sample along the normal to the surface, as well as a 
magnetic field applied in the same direction has been adopted for the XAS/XMCD studies. All measurements have been taken at room temperature in a magnetic field of 0.1-0.25 T with field switching between positive and negative field values at each photon energy value.

All samples have shown bulk ferromagnetism and $\mathrm{T}_{\mathrm{C}}$ above room temperature and a quasi-linear decreasing dependence of the $\mathrm{T}_{\mathrm{C}}$ for increasing $\mathrm{Cl}$ and $\mathrm{Br}$ doping levels in agreement with previous results (Fig.1 and inset) $(13,14)$. The $T_{C}$ decrease can be explained in terms of the reduction of the superexchange interaction between $\mathrm{Cr}$ ions and the increase in the lattice parameter $(14,17)$ once Se anions are substituted by $\mathrm{Cl}$ and $\mathrm{Br}$.

XAS spectral lineshapes provide information about the valence states of $\mathrm{Cr}$ and Cu cations. In Figure $2 \mathrm{Cr} L_{2,3}$ XAS spectra for increasing doping levels of $\mathrm{Cl}$ (a) and $\mathrm{Br}$ (b) doping agents are shown. In order to assess the presence of $\mathrm{Cr}^{3+}$ and $\mathrm{Cr}^{4+}$ cations, we have also plotted the $\mathrm{Cr}$ lineshapes for $\mathrm{Cr}_{2} \mathrm{O}_{3}\left(\mathrm{Cr}^{3+}\right), \mathrm{CrO}_{2}\left(\mathrm{Cr}^{4+}\right)$ (18) (c) and the spinel $\mathrm{CuCr}_{2} \mathrm{O}_{4}\left(\mathrm{Cr}^{3+}\right)$ for comparison (19). The lack of energy shift as a function of doping level and doping agent suggests a very similar electronic configuration for the $\mathrm{Cr}$ ions in all single crystals. A detailed analysis of the $\mathrm{Cr} L_{3}$ edge reveals three main peaks in all samples. By comparing them in energy position and relative intensity to the ones of $\mathrm{Cr}_{2} \mathrm{O}_{3}$ and $\mathrm{CrO}_{2}$, it is clear that a $\mathrm{Cr}^{3+}$ electronic configuration is more representative of our $\mathrm{Cr}$ ions. This conclusion is also confirmed at the $\mathrm{Cr} L_{2}$ edge. There the XAS lineshape for a $\mathrm{Cr}^{3+}$ electronic configuration shows a double feature with the first peak more intense than the second one as compared to that of a $\mathrm{Cr}^{4+}$ configuration where the situation is reversed. Finally, we compare our data to that of spinel structure $\mathrm{CuCr}_{2} \mathrm{O}_{4}$ known to have a $\mathrm{Cr}^{3+}$ electronic configuration (19). Except for the effects of different 
spin-orbit splitting due to the $\mathrm{Cr}$ ions being in an O octahedral structure as opposed to a Se octahedral one, our chalcogenide spinel spectra match very well to that of the oxide spinel with respect to all three main peaks at the $L_{3}$ edge and the double peak at the $L_{2}$ edge. It should be also noted that exposure to air does not affect the Cr XAS lineshape, and hence its electronic configuration, as shown by the similarities in the spectra of Cldoped samples ( $\mathrm{x}=0.0079$ and 0.0248$)$ that were kept in air and in an argon environment to minimize oxidation effects. The robustness to air exposure is in agreement with previous work (14) where doped single crystals have been found to be very stable in air.

In Figure 3, we show the XMCD data for different halogen doping agents at the Cr $L_{2,3}$ edges. Independent of dopant type, dopant concentration and exposure to air, the predominantly $\mathrm{Cr}^{3+}$ electronic configuration suggested by the XAS spectra is reflected also in the identical lineshape of the XMCD signals, thus suggesting a common magnetic configuration for $\mathrm{Cr}$ ions in all of our samples. Our findings seem to support the Goodenough model where the Cr ions are only in a 3+ valence state; however, it has to be pointed out that a small $\mathrm{Cr}^{4+}$ contribution cannot be totally ruled out given the similarities of the XAS spectra of the two valence states.

A detailed analysis of the $\mathrm{Cu} L_{2,3}$ XAS lineshapes in ref. [9] by J. B. Goedkoop et al. shows that $\mathrm{Cu}$ XAS spectra exhibit more dramatic changes as a function of the electronic configuration compared to the Cr XAS spectra. We observe similar effects in Figure 4(a) where the $\mathrm{Cu} L_{2,3}$ XAS spectra for different halogen doping type and air exposure are shown. The $\mathrm{Cu} L_{2,3}$ XMCD signals (Fig. 4 (b)) have been used to align the XAS spectra in energy. Based on the work of Noh et al. (11), we can attribute the three main peaks at the $L_{3}$ edge to three different contributions. The first one at lower photon 
energy $(\mathrm{E}=930.9 \mathrm{eV})$ can be assigned to $\mathrm{CuO}$ contamination due to the inevitable air exposure of all our single crystals. Although we have not been able to develop a procedure to expose clean surfaces during the measurements, such surface contamination has proved not to affect the electronic and magnetic properties of our samples as seen in Figures 2-4 and noted by Noh et al. (11). The second peak at E=932.0 eV is not always clearly visible but its presence is detected through the lower energy $(E=932.0 \mathrm{eV})$ dichroism signal and can be assigned to the $\mathrm{Cu}^{2+}$ valence state. It is important to point out that the detection of the XMCD signal at $\mathrm{Cu} L_{2,3}$ is already by itself an evidence of the presence of $\mathrm{Cu}$ ions with an unfilled $3 d$ shell, i.e. $\mathrm{Cu}^{2+}$. The third peak at $\mathrm{E}=933.7 \mathrm{eV}$ only represents the $\mathrm{Cu}^{+}$valence state according to Noh et al. Based on these peak assignments, we can conclude that all our samples show both $\mathrm{Cu}^{+}$and $\mathrm{Cu}^{2+}$ valence states with the same lineshape for the XMCD signal independent of doping level and doping agent.

The XMCD signal of the $\mathrm{Cu} L_{2,3}$ edges as seen in Figure 4(b) is indicative of magnetism associated with the $\mathrm{Cu}$ ions. Since $\mathrm{Cu}^{+}$ions with a full $3 d$ shell should not exhibit any magnetism, the XMCD signal is attributed to the $\mathrm{Cu}^{2+}$ ions. The XMCD feature at $\mathrm{E}=933.7 \mathrm{eV}$ suggests that the $\mathrm{Cu}^{2+}$ contribution to the $\mathrm{Cu}$ XAS signal extends beyond the peak assigned to $\mathrm{Cu}^{2+}$ valence by Noh et al.. A comparison of the $\mathrm{Cr}$ and $\mathrm{Cu}$ XMCD signal polarity reveal that the $\mathrm{Cu}$ moments are aligned antiparallel to the $\mathrm{Cr}$ moments. This magnetic configuration agrees well with band structure calculations of $\mathrm{CuCr}_{2} \mathrm{Se}_{4}(1)$.

In summary, we directly probed the electronic configuration of single crystals of $\mathrm{CuCr}_{2} \mathrm{Se}_{4-\mathrm{x}} \mathrm{Y}_{\mathrm{x}}$ with $\mathrm{Y}=\mathrm{Br}, \mathrm{Cl}$ via element and chemical specific XAS and XMCD in order 
to shed light on the long standing controversy regarding the electronic and magnetic state of $\mathrm{CuCr}_{2} \mathrm{Se}_{4}$. Our findings are in agreement with bulk magnetization studies by Miyatani et al. on $\mathrm{CuCr}_{2} \mathrm{Se}_{4-\mathrm{x}} \mathrm{Br}_{\mathrm{x}}$ (14) and with XAS/XMCD studies by Noh et al. on $\mathrm{CuCr}_{2-\mathrm{x}} \mathrm{Ti}_{\mathrm{x}} \mathrm{Se}_{4}$ (11). The XAS results from $\mathrm{Cl}$ and $\mathrm{Br}$ doped samples suggest a combination of $\mathrm{Cr}^{3+}, \mathrm{Cu}^{+}$ and $\mathrm{Cu}^{2+}$ electronic states. XMCD results reveal a magnetic moment associated with the $\mathrm{Cu}^{2+}$ ions that are antiparallel to the $\mathrm{Cr}^{3+}$ ions. Together the XAS and XMCD results show that the electronic and magnetic state of $\mathrm{CuCr}_{2} \mathrm{Se}_{4}$ cannot be described by either the Lotgering or Goodenough model.

We would like to acknowledge J. M. Iwata for providing the $\mathrm{CuCr}_{2} \mathrm{O}_{4}$ reference sample. The Advanced Light Source is supported by the Director, Office of Science, Office of Basic Energy Sciences, of the U.S. Department of Energy under Contract No. DE-AC02$05 \mathrm{CH} 11231$. 


\section{References}

1) J. S. Bettinger, R. V. Chopdekar, M. Liberati, J. R. Neulinger, M. Chshiev, Y. Takamura, L. M. B. Alldredge, E. Arenholz, Y. U. Idzerda, A. M. Stacy, W. H. Butler and Y. Suzuki, J. Magn. Magn. Mater. 318, 65 (2007)

2) V. N. Antonov, V. P. Antropov, B. N. Harmo, A. N. Yaresko and A. Ya. Perlov, Phys. Rev. B 59, 14552 (1998)

3) C. Colominas, Phys. Rev. 153, 558 (1966)

4) M. Robbins, H. W. Lehmann and J. G. White, J. Phys. Chem. Solids 28, 897 (1966)

5) F. K. Lotgering and R. P. van Stapele, Solid State. Comm. 5, 143 (1967); F. K. Lotgering and R. P. van Stapele, J. Appl. Phys. 39, 417 (1968)

6) J. B. Goodenough, Solid State. Comm. 5, 577 (1967)

7) P. R.Locher, Solid State Comm. 5, 185 (1967)

8) A. W. Sleight, Mat. Res. Bull. 2, 1107 (1967)

9) Klaus Baberschke, Physica Scipta T115, 49 (2005); M. Grioni, J. B. Goedkoop, R. Schoorl, F. M. F. de Groot, J. C. Fuggle, F. Schafers, E. E. Koch, G. Rossi, J.-M. Esteva and R. C. Karnatak, Phys. Rev. B 39, 1541 (1989); S. W. Han, J.-S. Kang, S. S. Lee, G. Kim, S. J. Kim, C. S. Kim, J.-Y. Kim, H. J. Shin, K. H. Kim, J. I. Jeong, B.-G. Park, J.-H. Park and B. I. Min, J. Phys. Condens. Matter 18, 7413 (2006)

10) A. Kimura, J. Matsuno, J. Okabayashi, A. Fujimori, T. Shishidou, E. Kulatov and T. Kanomata, Phys. Rev. B 63, 224420 (2001)

11) H.-J. Noh, J.-S. Kang, S. S. Lee, G. Kim, S.-W. Han, S.-J. Oh, J.-Y. Kim, H.-G. Lee, S. Yeo, S. Guha and S.-W. Cheong, Europhys. Lett. 78, 27004 (2007)

12) A. Deb, M. Itou, V. Tsurkan and Y. Sakurai, Phys. Rev. B 75, 24413 (2007) 
13) O. Yamashita, H. Yamauchi, Y. Yamaguchi and H. Watanabe, J. Phys. Soc. Jpn. 47, 450 (1979)

14) K. Miyatani, K. Minematsu, Y. Wada, F. Okamoto, K. Kato and P. K. Baltzer, J. Phys. Chem. Solids 32, 1429 (1971)

15) Janell R. Neulinger, PhD Thesis, UC Berkeley, 2006

16) A. T. Young, E. Arenholz, J. Feng, H. Padmore, S. Marks, R. Schlueter, E. Hoyer, N.

Kelez and C. Steier, Surf. Rev. Lett. 9, 549 (2002)

17) A.W. Sleight and H.S. Jarrett, J. Phys. Chem. Solids 29, 868 (1967)

18) Yu. S. Dedkov, A. S. Vinogradov, M. Fonin, C. Konig, D. V. Vyalikh, A. B. Preobrajenski, S. A. Krasnikov, E. Yu. Kleimenov, M. A. Nesterov, U. Rudiger, S. L. Molodtsov and G. Guntherodt, Phys. Rev. B 72, 60401 (2005)

19) Jodi M. Iwata, unpublished data 
Figure captions:

Figure 1: (color online) $\mathrm{T}_{\mathrm{C}}$ of $\mathrm{CuCr}_{2} \mathrm{Se}_{4-\mathrm{x}} \mathrm{Y}_{\mathrm{x}}$ single crystals as a function of increasing doping level and different halogen doping agents $(\mathrm{Y}=\mathrm{Cl}, \mathrm{Br})$. The inset shows a hysteresis loop at $300 \mathrm{~K}$ for a $\mathrm{CuCr}_{2} \mathrm{Se}_{4-\mathrm{x}} \mathrm{Br}_{\mathrm{x}}$ single crystal with $\mathrm{x}=0.3932$.

Figure 2: (color online) XAS at $\mathrm{Cr} L_{2,3}$ of $\mathrm{CuCr}_{2} \mathrm{Se}_{4-\mathrm{x}} \mathrm{Y}_{\mathrm{x}}$ single crystals as a function of increasing doping level for $\mathrm{Cl}(\mathrm{a})$ and $\mathrm{Br}$ (b) halogen doping agent. Reference spectra for $\mathrm{Cr}^{3+}$ and $\mathrm{Cr}^{4+}$ (c) are taken from reference 18. Reference spectra of $\mathrm{Cr}^{3+}$ for $\mathrm{CuCr}_{2} \mathrm{O}_{4}$ spinel plotted in a) and b) is from reference 19.

Figure 3: (color online) XMCD at $\mathrm{Cr} L_{2,3}$ edges for $\mathrm{CuCr}_{2} \mathrm{Se}_{4-\mathrm{x}} \mathrm{Y}_{\mathrm{x}}$ single crystals as a function of different doping halogen agent ( $x=0.3932$ for $\mathrm{Br}$ doping, $\mathrm{x}=0.0248$ for $\mathrm{Cl}$ doping) and air exposure ( $\mathrm{x}=0.0248$ for $\mathrm{Cl}$ doping and kept in $\mathrm{Ar}$ ).

Figure 4: (color online) (a)) XAS and (b) XMCD at $\mathrm{Cu} L_{2,3}$ edges for $\mathrm{CuCr}_{2} \mathrm{Se}_{4-\mathrm{x}} \mathrm{Y}_{\mathrm{X}}$ single crystals as a function of different doping halogen agent ( $\mathrm{x}=0.3932$ for $\mathrm{Br}$ doping, $\mathrm{x}=0.0248$ for $\mathrm{Cl}$ doping) and air exposure ( $\mathrm{x}=0.0248$ for $\mathrm{Cl}$ doping and kept in Ar). 


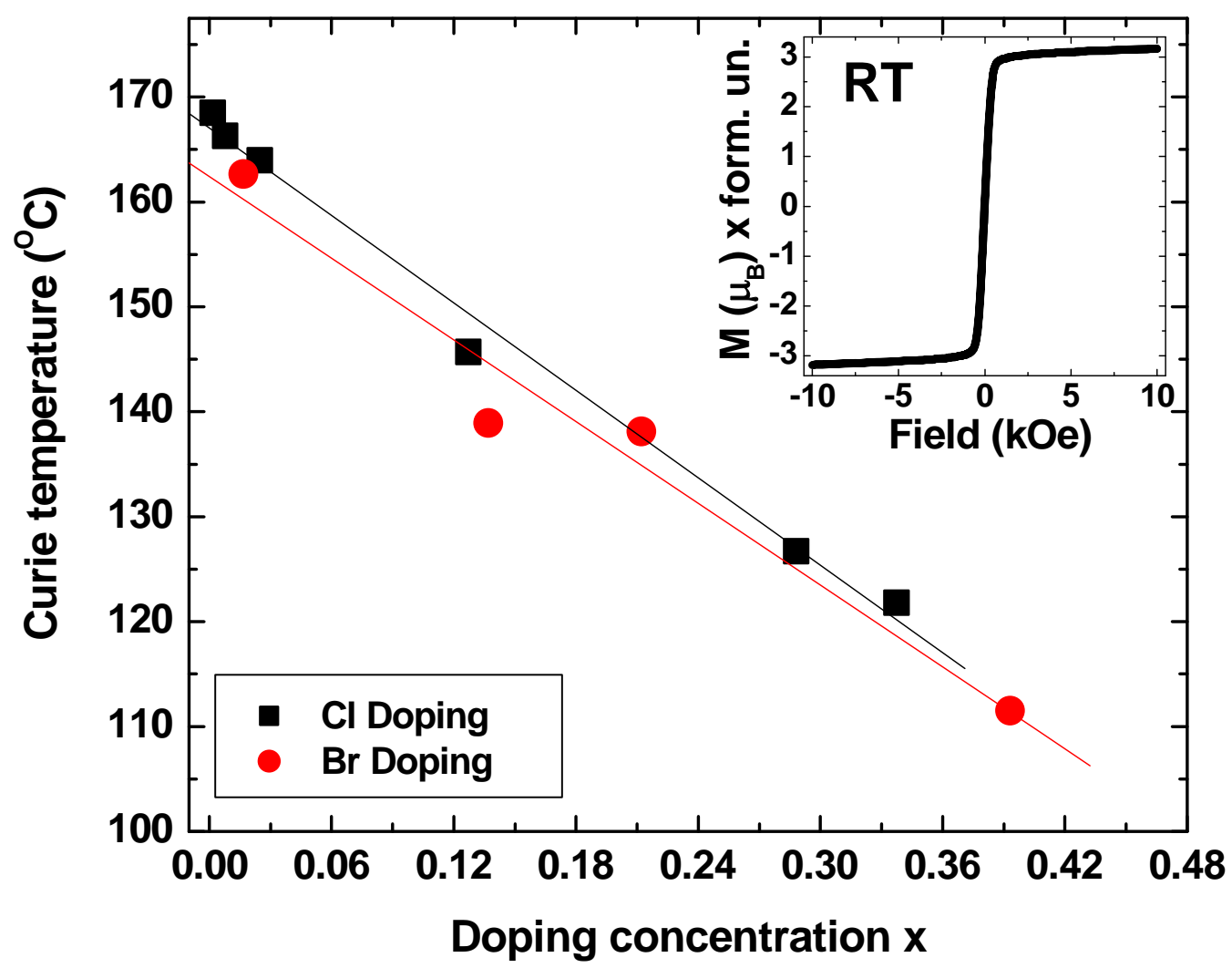

FIGURE 1 

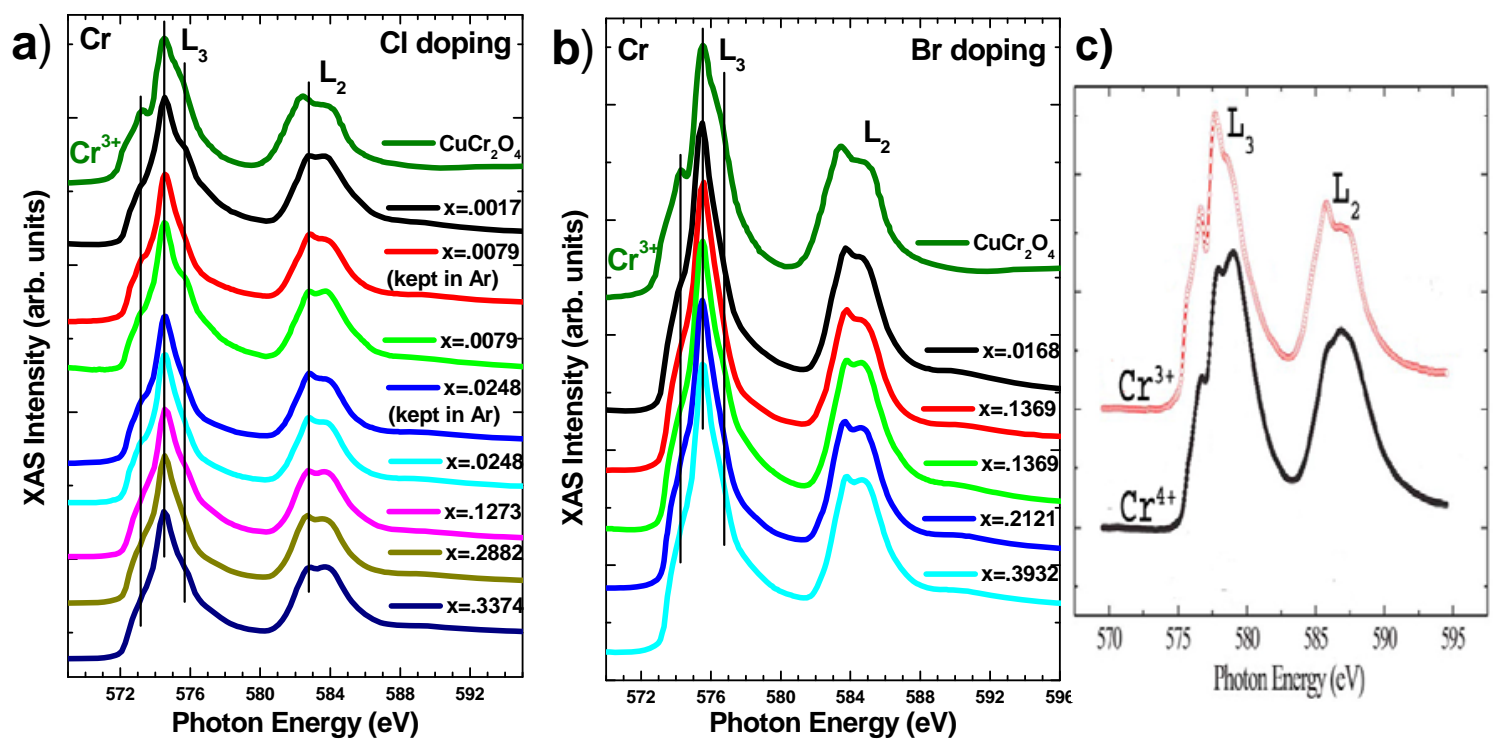

\section{FIGURE 2}

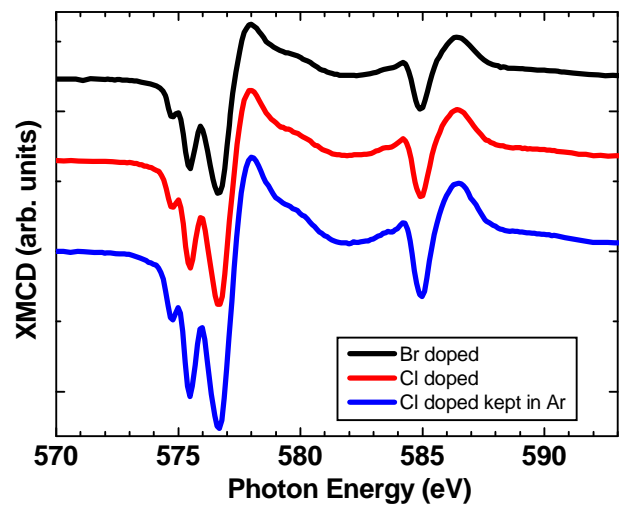

FIGURE 3 


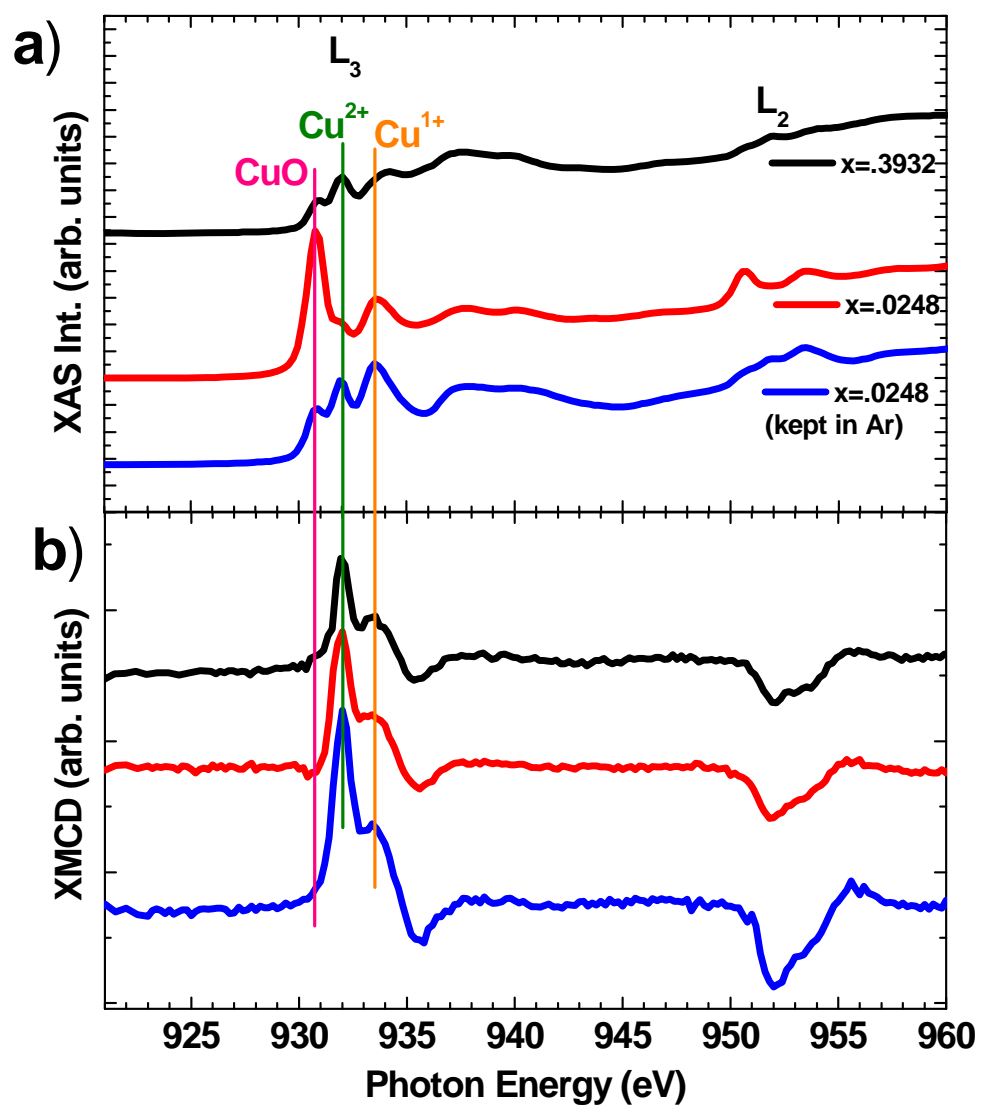

FIGURE 4 